\title{
Voting-based License Plate Location
}

\author{
Yong Zhao, Yule Yuan, Member, IEEE, Subin Bai, Kai Liu, Wei Fang
}

\begin{abstract}
This paper introduces a novel framework which uses all kind of features to elect a license region from an image. The proposed method consists of four major parts: image enhancement, the first round of voting to get the candidate blocks, boundary adjustment, and the second round of voting to get the real plate image. Experiments show that the average accuracy of locating license plate is $\mathbf{9 8 . 2} \%$ under the experiment databases, which were taken from real scene including different orientation variations, daytime and night time.
\end{abstract}

\section{INTRODUCTION}

$\mathrm{N}$ OWADAYS, traffic congestion has been increasing worldwide as a result of increased population growth, motorization and changes in the way of people's travel. The ITS (Intelligent Transport System) technologies have gotten so much attention and turned to be an important research issue to solve the traffic congestion. One type of ITS technology is the LPR (License Plate Recognition). The ITSs rely heavily on the LPR system, because the license plate remains as the principle vehicle identifier.

Typically, a LPR system mainly consists of three parts; that is the LPL (License Plate Location), the character segmentation, and the character recognition. Among them, the license plate search is considered as the most crucial step in the license plate recognition system, which directly effects robustness and accuracy of the LPR system. To make the location process accuracy and robust, many challenges must be overcome, such as the contaminated plates, wristed plates. And, if complex backgrounds are also involved, locating license plates can become quite a challenge. The aim of this paper is to improve the location accuracy in complex scene.

In the past, researchers have found a number of techniques to location the license plate. Park trained a neural network to locate license plate [1]. Rodolfo and Stefano et al [2] devised a method based on vector quantization (VQ) to process the images, which makes it possible to perform superior picture compression for archival purposes and to support effective location at the same time. J.W. Hsieh, S.H. Yu, and Y.S. Chen el at [3] based on morphology method, which can significantly reduce the number of candidates, and thus speeds up the subsequent plate recognition. A fast license plate extraction method based on the vertical density map proposed in [4] on

Yong Zhao is with the faculty of Shenzhen Graduate School, Peking University, Shenzhen, CO 518055, China (e-mail: zhaoyong@pkusz.edu.cn). complex background. But the short- coming of the algorithm is in falling to locate the license plate that is badly deficient. Bai and Liu proposed a hybrid license plate extraction method based on edge statistics and morphology. By using this method a notable improvement has been described in [5]. Zhu et al. [6] Wei et al. [7], and Chang el al. [8] used the color features to locate license plate, but these approaches are not robust enough to different environment. Because the automatic license plate contains rich edge information, many methods based on it were proposed, such as Ming et al. [9], and Zheng el al. [10]. Jia et al. [11] used the Mean Shift algorithm to segment color images into candidate regions and subsequently classified as plate or not. In the work of Donoser et al. [12] proposed an efficient algorithm based on Maximally Stable Extremal Region (MSER) detection without the need of any learning scheme. Chen et al. [13] used the salient features to locate the plate. Recently, image statistical analysis is concerned, license plate detected based on a probabilistic model in the work [14]. In order to dealing with the high resolution image quickly in plate location algorithm, the method of [15] introduced a scanning method that is Operator Context Scanning (OCS) to speed up the image scanning process.

Although much scientific work has focused on detecting license plates form images, surprisingly little work has been done on integrating a scheme to locate a plate. As we known, it is difficult to accuracy locate a plate under different weather and lighting conditions (cloudy, sunny, daytime, night time). In this paper, we further research the subject of license plate detection. The process of presidential election inspired us to propose the scheme. The difference is that our president is just a license plate. In the election process, we use a variety of features, such as vertical edge feature, the density of edge map, projection features and so on. Fig. 1 shows the overview of the proposed method, which consists of four major parts: image enhancement, the first round of voting to get the candidate blocks, border adjustment, and the second round of voting to get the real plate image.

The main contribution of this paper is a novel framework which uses all kind of features to elect a license region from an image. Experiment shows that our scheme performs effectively to detect license plate from images in a broader range of conditions, including shadows, dirt from bumpers, sun reflections in the plate region. 


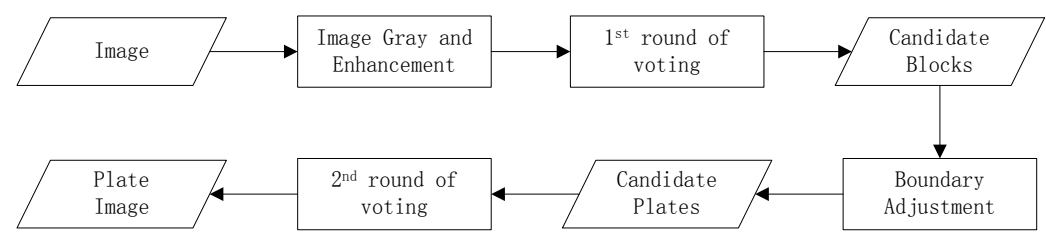

Fig.1. Flowchart for the license plate location system

\section{The Details of License Plate Location}

\section{A. Image Gray and Enhancement}

For some low contrast images, it is necessary to enhance them before plate location. The edge details of an image will be obscured when the image is under-exposed or overexposed. We can usually enhance the details of the image, if we extend the grayscale. We can use the eq. (1) to achieve this purpose.

$$
I_{x, y}^{\prime}=\left\{\begin{array}{l}
\frac{c}{a} * I_{x, y}, I_{x, y}<a \\
\frac{(d-c)}{(b-a)} *\left(I_{x, y}-a\right)+c, a \leq I_{x, y}<b \\
\frac{(255-d)}{(255-b)} *\left(I_{x, y}-b\right)+d, I_{x, y} \geq b
\end{array}\right.
$$

Where $I_{x, y}$ is the source image pixel gray value and it's gray-scale range is $[a, b]$. The expected dynamic range of enhanced image $I_{x, y}^{\prime}$ is [c, d]. In our system, the value of $c, d$ is 30 and 225 respectively.

\section{B. The First Round of Voting}

Firstly, we split the image to many block candidates. The image and the blocks size is $\mathrm{W} \times \mathrm{H}$, and $\mathrm{N} \times \mathrm{N}$ respectively. Here $\mathrm{N}$ is 15 . In the first round of voting, only by meeting the equation (2) can be a candidate block (CB), which means the $\mathrm{CBF}$ equals 1 . Here, $\alpha$ is the coefficient of the VT.

$$
C B F[Y][X]=\left\{\begin{array}{l}
1, \text { block_mean_sobel } \geq \alpha \times \mathrm{VT} \\
0, \text { block_mean_sobel }<\alpha \times \mathrm{VT}
\end{array}\right.
$$

Where, $\mathrm{CBF}$ is a candidate block flag array for each $\mathrm{N} \times \mathrm{N}$ blocks. The range of the value of $\mathrm{X}$ and $\mathrm{Y}$ is from 0 to $\mathrm{W} / \mathrm{N}$ or $\mathrm{H} / \mathrm{N}$ respectively. The symbol 'block_mean_sobel' means the mean values of the corresponding block that get from the sobel image. The values of this parameter follow the pattern of equation (3).

$$
\text { block_mean_sobel }=\frac{1}{N \times N} \sum_{j=b x_{0}}^{b x_{0}+N} \sum_{i=b y_{0}}^{b y_{0}+N} \operatorname{sobel}[i][j]
$$

Where 'sobel' represents the values from sobel image, which is the enhanced image of the input image after vertical sobel operation (in Eq. (4)). The sobel image is shown in Fig.2.

$$
\text { sobel }[x][y]=\left[\begin{array}{ccc}
-1 & 0 & 1 \\
-2 & 0 & 2 \\
-1 & 0 & 1
\end{array}\right] \otimes I[x][y]
$$

Where, VT is the parameter of the voting threshold value. The values of this parameter can be gotten from Eq. (5).

$$
V T=\frac{1}{(W \times H) /(N \times N)} \sum_{x=0}^{W} \sum_{y=0}^{H} \operatorname{sobel}[x][y]
$$

In the first round of voting, most of the candidate blocks are removed. Next, the remained candidate blocks of the image undergo connected component analysis, removal of some candidate blocks in isolation. After these steps, the result is as shown in Fig.3 (a).

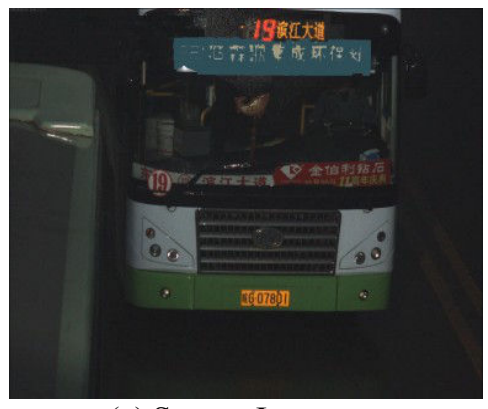

(a) Source Image

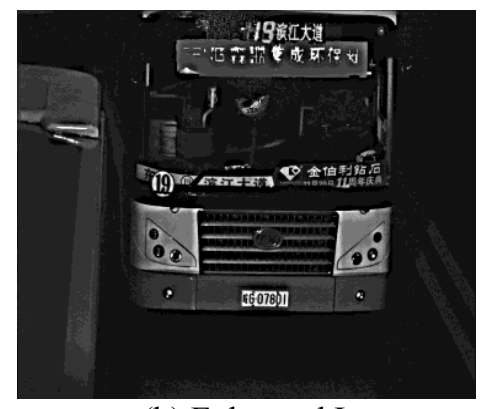

(b) Enhanced Image

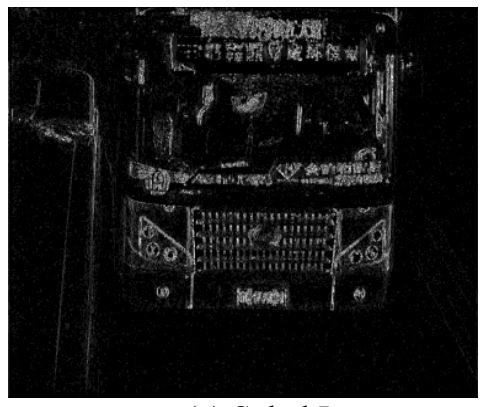

(c) Sobel Image

Fig. 2. Enhacement and vertical sobel operator experiment results can be used for location license plate. 


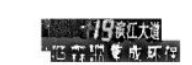

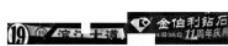

[700]

[60780]

(a) Image block elected
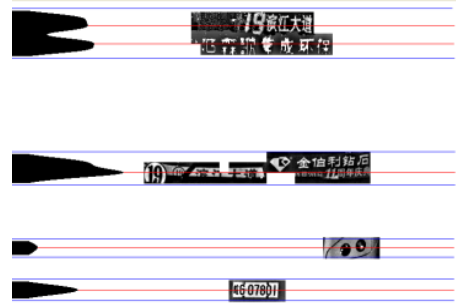

(b) Horizontal boundary

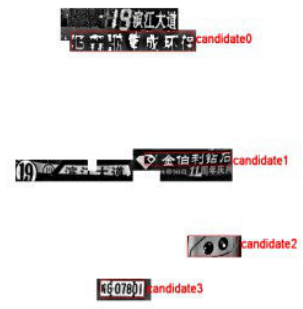

(c) Vertical Boundary

Fig.3. The first round bock voting experiment results and the boundary adjustment for these candidate blocks.

\section{Boundary Adjustment}

In this subsection we discuss the boundary adjustment. The third step of license plate location is the adjustment of the boundary of the plate. Then, we use the horizontal projection to determine the horizontal boundaries of the license plate and the vertical projection to determine the vertical boundaries of the plate. Fig. 3 (b, c) shows the adjustment effect. The process of boundary adjustment is step by step. After determine the horizontal and vertical boundaries, we need to merge the blocks within the frame. As shown in Fig.3, (c) represent the four candidate plates after the above produce. We marked them in the picture with 'candidate0', 'candidate1', 'candidate2', and 'candidate3' respectively. The next section will determine the real plate from the four candidate regions.

\section{The Second Round of Voting}

Some typical Chinese license plates are shown in Fig.4, and the figure comes from the standard of vehicle plate in the People's Republic of China. There are several feathers [16] of license plate which can be made use of in the algorithm proposed as prior knowledge.

(1) There are four kinds of Chinese license plates: white characters on a blue background, white characters on a black back ground, black characters on a yellow background and black or red characters on a white background. And different color types of plate indicate different vehicle types.

(2) In each license plate, there must be seven characters.

(3) The outline size of license plate is $440 * 140\left(\mathrm{~m}^{2}\right)$ and ratio of width to height is about 3 . The size of character is $45^{* 90}$ $\left(\mathrm{m}^{2}\right)$ and ratio of height to width is about 2.

(4) The interval of adjacent symbol usually is $12 \mathrm{~mm}$. But the interval of the second symbol to the third one is $34 \mathrm{~mm}$, which is much large than that of others.

The basic idea of voting a license plate from the given candidate plates is similar with presidential election process.
According to the similar characteristics of the real plates, the voting rules for the license plate are defined as follows:

- The number of vertical projection peaks of the candidate is between 7 to 15 . As we all known, the Chinese plate usually has at least 7 peaks. Taking into account the reasons for the border and noise, the number range is from 7 to 15 . It is a very import feature to decide a candidate region will be or not to be a plate. We set the weight of the peaks is 3 .

- It should have the largest texture complexity. Here we use Eq. (6) to measure it. The weight of this rule is 2 . Where CMS means mean value of the candidate sobel values. The parameter $\left(\mathrm{x}_{0}, \mathrm{y}_{0}\right)$ is the starting point of the candidate and 'cw', 'ch' is the candidate region's width and height respectively.

$$
C M S=\frac{1}{(c w \times c h)} \sum_{i=x_{0}}^{x_{0}+c w} \sum_{j=y_{0}}^{y_{0}+c h} \operatorname{sobel}[i][j]
$$

Aspect ratio should be between 2.5 to5. The value can get from the Eq. (7). The weight of this rule is 1.

$$
C R=\frac{c w}{c h}
$$

- The candidate region's background color should be one of the colors (blue, yellow, white, black) of a plate's background. The weight of this rule is 1 .

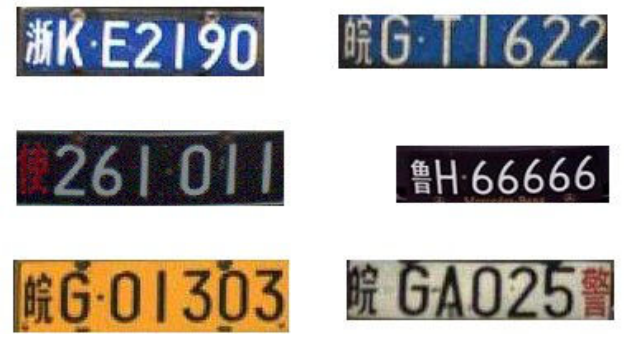

Fig.4. Some Chinese Plates 
What should be noted that here we only consider the Chinese plates. Finally, we vote by the rules that define above. The voting result is clearly shown in Table I. From these test data, we can draw a conclusion that the candidate 4 is the real plate which we want to locate.

TABLE I

CANDidate Region Voting EXamined

\begin{tabular}{cccclc}
\hline Name & Peaks & CMS & CR & Color & Votes \\
\hline \hline candidate0 & 17 & 177 & 8.1 & Blue & 1 \\
candidate1 & 12 & 184 & 8.4 & Red & 3 \\
candidate 2 & 4 & 115 & 3.2 & White & 2 \\
candidate3 & 13 & 352 & 3.7 & Yellow & 7 \\
\hline
\end{tabular}

\section{EXPERIMENTAL RESULTS}

We evaluated our proposed license plate location method on image dataset which was captured from a real highway ticketing stations at different lighting condition using Basler ${ }^{\mathrm{TM}}$ industrial cameras. Experiments have been implemented to test the performance and accuracy of the proposed system. 5436 color images are been used for experiment. Most of the images processed have a resolution $1624 \times 1232$ and therefore the plates have an average size of 165 x 50 pixels in the dataset. The minimum plate resolution that the system can recognize is about $70 \times 20$.

The personal desktop computer used to conduct the experiment is a Pentium 4 at $2.5 \mathrm{GHZ}$ with $3 \mathrm{G}$ RAM. Experimental results show that only 96 images could not be located correctly. In the 96 images to be detected error, most plates of them were badly damaged or be partially obscured. The accuracy of detecting vehicle license plate is above $98 \%$. The processing time of one color image is about $230 \mathrm{~ms}$. It is very fast to detect license for HD pictures. It is because that most of the blocks eliminated in the first round of voting.

\section{CONCLUSION}

This paper presented a novel framework which uses all kind of features to elect a license region from an image. The proposed method consists of four major parts: image graying and enhancement, the first round of voting to get the candidate blocks, horizontal and vertical boundary adjustment, and the second round of voting to locate the real plate image. Experiments show that the average accuracy of locating license plate is over $98 \%$ under the experiment databases, which were captured under a variety of environmental conditions. Due to the first round of voting removed most of the blocks in the image, the overall processing time is only used $230 \mathrm{~ms}$ to deal with the HD image (1624 x 1232). Experimental results demonstrate the great robustness and efficiency of the license plate location method.

Future work should include the incorporation of our character segmentation and recognition module.

\section{ACKNOWLEDGMENT}

The authors gratefully acknowledge the Mr. P. Guo of the Landun Tec. Company provides all the test dataset for this paper.

\section{REFERENCES}

[1] S.H. Park, K.I. Kim, K. Jung, and H.J. Kim, "Locating car license plates using neural networks," Electron. Lett.35, 1999, pp.1475-1477.

[2] Rodolfo Z., and Stefano R., "Vector quantization for license plate location and image coding," IEEE Trans. on Industrial Electronics, vol.47, no.1, Feb.2000, pp.159-167.

[3] J.W. Hsieh, S.H. Yu, and Y.S. Chen, "Morphology-based License Plate Detection from Complex Scenes," Pattern Recognition, 2002.

[4] H. Bai, J. Zhu, and C. Liu, "A Fast License Plate Extraction Method on Complex Background," Proceedings of Intelligent Transportation Systems Conference, IEEE, vol.2, 2003, pp.985-987.

[5] H. Bai, C. Liu, "A hybrid License Plate Extraction Method based on Edge Statistics and Morphology”, IEEE ICPR’04, 2004, pp.831-834.

[6] W.G. Zhu, G.J. Hou, "A study of locating vehicle license plate based on color feature and mathematical morphology," Proceedings of International Conference on Signal Process., vol.1, 2002, pp.748-751.

[7] W. Wei, M.J. Wang, and Z.X. Huang, "An automatic method of location for number-plate using color features," Proceedings of International Conference on Image Process., vol.1, 2001, pp. 782-785.

[8] S.L. Chang, L.S. Chen, Y.C. Chung, and S.W. Chen, "Automatic License Plate Recognition," IEEE Trans. On Intelligent Transportation Systems. Vol.5, No.1, Mar. 2003, pp.42-53.

[9] G.H. Ming, A.L. Harvey, and P. Danelutii, "Car number plate detec- tion with edge image improvement," Preceding of Internat. Symp. On Signal Process and its Applications, vol.2, 1996, pp.597-600.

[10] D. Zheng, Y. Zhao, and J. Wang, "An efficient method of license plate location," Pattern Recognition Letters, vol.26, no.5, Nov. 2005, pp.2431-2438.

[11] W. Jia, H. Zhang, X. He, and M. Piccardi, "Mean shift for accurate license plate location," In ITSC. Proceedings of the IEEE Conference on Intelligent Transportation Systems, 2005, pp.566-571.

[12] Donoser Michael, A. Clemens, and B. Horst, "Detecting, Tracking and Recognizing License Plates," Lecture Notes in Computer Science, vol.4844 LNCS, no part 2, Computer Vision- ACCV- $8^{\text {th }}$ Asian Conference on Computer Vision, 2007, pp.447-456.

[13] Z. Chen, G. Wang, J. Liu, and C. Liu, "Automatic License Plate Location and Recognition Based on Feature Salience," International Journal of Computational Cognition. Vol.5, No.2, Jun. 2007, pp. 3781-3785.

[14] A.H. Rami, and C. Subhash, "License plate localization based on a probabilistic model," Machine Vision and Applications, vol. 21, no.3, Apr. 2010, pp.319-330.

[15] Ioannis Giannoukos, C. N. Anagnostopoulos, V. Loumos, and E. Kayafas, "Operator context scanning to support high segmentation rates for real time license plate recognition," Pattern Recognition 43, 2010, pp.3866-3878.

[16] Feng Yang, Zheng Ma, and Mei Xie, "A Novel Approach for License Plate Character Segmentation," $1^{\mathrm{ST}}$ IEEE Conference on Industrial Electronics and Applications, Singapore, pp.1-6, 2006. 